\section{Experimente wagen!}

_ Gestern kehrte ich zurück von einer Veranstaltung mit einem völlig neuen Konzept, das ich als bisher einzigartig erlebt habe. Eine Ergotherapeutin hatte ein gemeinsames Symposium für Ergotherapeuten, Lehrer, Mediziner, Psychologen, Betroffene und interessierte Laien auf die Beine gestellt: die ersten ADHS-Tage in Hannover. Ein Experiment, mit dem unsere Kollegin Erfolg hatte. 350 Teilnehmer folgten Britta Winters Ruf und diskutierten über die vielfältigen Behandlungsansätze bei Kindern mit ADHS. Das Besondere: Die Organisatorin hatte es mit ihrem Programm geschafft, wirklich alle, die mit den betroffenen Kindern zu tun haben, an einem Ort zu versammeln und bot dadurch einen runden Tisch im XXL-Format an.

_ Ebenfalls ein Experiment war das Ergoforum Demenz, als es vor etwa zwei Jahren zum ersten Mal in Stuttgart stattfand. Ein Forum für informellen Austausch und regionale Vernetzung. Auch dieses Konzept trug: Aufgrund der positiven Resonanz entschied sich Sylvia Kern, Geschäftsführerin der Alzheimer Gesellschaft Baden-Württemberg, die Organisation ein zweites Mal in die Hand zu nehmen. Mit Erfolg! Das Treffen im Februar bot den circa 40 Teilnehmern zahlreiche neue Anregungen. Sie identifizierten ähnliche Sorgen und Nöte und waren sich daher einig, sich künftig regelmäßiger in Arbeitsgruppen zu treffen und stärker zu vernetzen (๑ Seite 38).

_ Aber nicht nur bei Veranstaltungen wird mit neuen Konzepten experimentiert. Ergotherapeuten engagieren sich zunehmend politisch und reichen beispielsweise Petitionen im Deutschen Bundestag ein. Sie betreiben Öffentlichkeitsarbeit über die Lokalpresse, stellen dort unser Berufsbild vor. Andere wiederum publizieren ihre Erfahrungen in Fachzeitschriften und geben sie auf diesem Weg an interessierte Kollegen weiter wie zum Beispiel Friederike Grill mit ihrem Artikel über Validation ab Seite 20. Oder sie bringen ihr Fachwissen in neuen Arbeitsfeldern ein wie Lorena Honold, die als Einzelintegrationsberaterin bei der Stadt Düsseldorf angestellt ist ( $\odot$ Seite 30 ).

_ All diese Puzzlestücke zeigen mir eines: Wir sind eine kreative Berufsgruppe mit großem Potenzial. Lassen Sie uns also weiterhin viele Experimente wagen, die uns als professionell denkende und handelnde Profession für andere sichtbar machen!

Ich wünsche Ihnen dabei viel Erfolg!

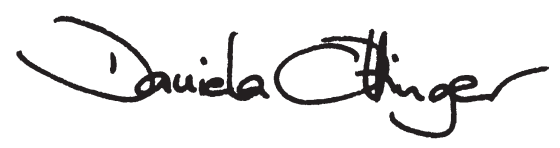

ZU GEWINNEN

Kursplatz

1 Kurs „Fingerspitzengefühl

für alle“

Seite 44

Bücher

4-mal „Ratgeber

Neuropsychologie“"

Seite 16

3 Hörbücher zum Thema

Validation

Seite 23 